\section{Questión}

Periodismo / Comunicación ISSN 1669.6581

La experiencia como problema en el fútbol

Pablo Fusetti

Question/Cuestión, Nro.70, Vol.3, diciembre 2021

ISSN: 1669-6581

URL de la Revista: https://perio.unlp.edu.ar/ojs/index.php/question/

IICom -FPyCS -UNLP

DOI: https//doi.org/10.24215/16696581e633

\title{
La experiencia como problema en el fútbol
}

\section{The experience as a problem in football}

Pablo Fusetti

Universidad Nacional de La Plata

Facultad de Humanidades y Ciencia de la Educación Instituto Superior de Formación Docente N84

Argentina

pablofusetti@gmail.com

https://orcid.org/0000-0002-6173-0408

\section{Resumen}

En el fútbol argentino, la experiencia se presenta como principio de autoridad supremo, convalidando las acciones de su orden, organizando y sosteniendo su estructura, y determinando la mayoría de decisiones institucionales. Colocar a la experiencia por sobre la teoría no solo es anular la atadura histórica que tiene la práctica y los sujetos, sino es la subestimación de lo novedoso. Esto constituye un problema del orden político en el fútbol, 
porque la experiencia no cuestiona, sino que reproduce el orden instituido. De este modo, el fútbol disminuye las probabilidades de ser alterado tanto en su organización política como en sus enunciados epistémicos. La idea de la siguiente presentación es detenerse aquí para problematizar la situación mencionada: el triunfo de la experiencia vivencial sobre la teoría, el vaciamiento con el saber teórico que ningunea algún cambio posible.

Palabras claves: Fútbol; experiencia; política.

\section{Abstract}

In the Argentinian football, the experience is presented as a supreme principle of authority, validating the actions of its order, organizing and supporting its structure, and determining the majority of institutional decisions. To place experience above theory is not only to nullify the historical ties between practice and subjects, but also to underestimate what is new. This contributes to create a political problem, because the experience does not question, but reproduces the instituted order. In this way, football reduces the chances of being changed/altered both in its political organization and epistemic statements. The main idea of the following presentation is to problematize the mentioned situation: the triumph of the experience over the theory, the emptying of the theoretical knowledge that does not make any possible change.

Keywords: Football; experience; politics.

\section{Inicio esquemático}

En la actualidad vemos instalado fuertemente un razonamiento: para enseñar un deporte debes haberlo vivenciado, practicado, de lo contrario, no estás apto para desempeñarte en esa tarea. También, vemos instalada aquella idea de presentar a alguna persona habilitada para hablar de fútbol -como jugador, como comentarista, como entrenador o dirigente, entre otros roles-, pero en donde esa habilitación se subsume únicamente su recorrido sensible por los distintos clubes. Estamos hablando de una absorción sensible del saber, la noción de que por haberla vivido "desde adentro", esta experiencia se convierte en atributo indiscutible para legitimar tanto la enseñanza, la gestión o simplemente tomar la 
palabra en el fútbol; nos referimos, al valor que se le da a la "vivencia" y su uso político como represión amable.

En este punto, resulta útil realizar una distinción conceptual entre Erlebnis y Erfahrug como dos posibles sentidos de experiencia. Michael Löwy se refiere a estos dos conceptos diferentes de experiencia tal y como aparecen en Benjamin, y describe a la Erfahrung como una "experiencia auténtica (...) fundada en la memoria de una tradición cultural e histórica-" (2005, p. 29); y la distingue de la Erlebnis como "vivencia inmediata".

En la constatación de Benjamin de la crisis de la experiencia, lo que está en crisis no es la vivencia (Erlebnis), sino la experiencia en el sentido de Erfahrung, aquella que, excediendo el ámbito personal o privado de la mera vivencia, resulta comunicable adquiriendo de ese modo un carácter colectivo o compartido (Staroselsky Tatiana, 2015, p. 4).

La vivencia la podríamos colocar fuertemente del lado individual, del plano empírico, algo inmediato que le sucede a una persona en un contacto sensorial continuo con los hechos, los cuales pasan y la atrapan en forma de adhesión. De este modo, las vivencias se van adhiriendo con las anteriores en un movimiento progresivo hacia el conocimiento. La experiencia, en cambio, tiene lugar en la memoria histórica, en una actualización colectiva de los hechos que rompe con lo continuo y monótono de los mismos. Es una interrupción del continuo, una exhibición a lo no conocido que se produce en el contacto con el lenguaje para reelaborar desde aquí otra conjunción al conocimiento, otra conjunción de nuestro pensamiento.

Ahora bien, muchas veces se dice que falta un "proyecto" en el fútbol, que cada director técnico viene, hace lo que quiere y puede, le va como le va, termina su conducción y luego viene otro y cambia todo, y así sucesivamente. Sin embargo, más que un proyecto, la carencia es con lo teórico. Uno de los problemas más grandes en relación a este tema consiste en que su configuración se construye desde la pura empírea, desde un armado de decisiones regidas por la inmediatez de los hechos. En otras palabras, se buscan respuestas en el 
contacto con el mundo real como si ahí hubiera algo que encontrar, algo que descubrir en el ejercicio de esa percepción sensible que los vestuarios, los entrenamientos, o los partidos en sí, sean ellos por si mismos lo que nos permitan acceder al conocimiento del fútbol.

En relación a esto, se expone el presente cuadro extraído del libro publicado en el año 2004 por Horst Wein "Futbol a la medida del niño", visibilizando el grado de importancia que se le atribuye al concepto de experiencia-vivencia para enseñar fútbol:

\section{FACTOAESI H A C QMADOS CONEA CAPACIDAD DEL FORMAOOF}

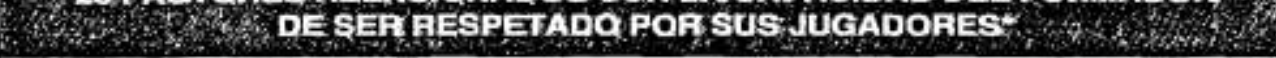

1. Experiencia previa como jugador y bxito.

2. Experiencia previa como entrenador y éxito.

3. Buena apariencia: vestido, torma fisica.

4. Adecuados hábitos de vida.

5. Buenos hábitos de trabajo: ajuste a los horarios, eficiencia.

6. Bien organizado: entrenamientos, reuniones, viajes, etc.

7. Buen comunicador: explica con claridad, sabe escuchar.

8. Disponibilidad: tiene siempre tiempo para ef jugador.

9. Conocimiento; demuestra conocimiento del juego, tanto de los aspectos técricos como de los tacticos.

10. Habilidad para ensenar: manifiesta capacidad para corregir errores tócnicos y tacticos.

11. Alta motivación: intensidad, compromiso, implicación.

12. Positivo: ánimo, enusiasmo, optimismo, elogios y refuerzos positivos.

13. Buen entrenador en el banquilio: realiza reajustes, analiza y reacciona, hace cambios y variaciones, según la marcha del partido.

14. Buen sentido del humor: ambiente distendido.

15. Buena capacidad de liderazgo: en el vestuario y durante el partido.

16. Buen nivel de autocontrol: control emocional, contagia calma y serenidad.

17. Deseo de mejorar: busca nuevos conocimientos, aprende, investiga. se autoevalúa.

18. Honesto y justo con los jugadores: no muestra favoritismos, es "duro pero justo".

19. Abierto a sugerencias: flexible, escucha las sugerencias de jugadores y ayudantes.

20. Muestra un verdadero interés por los jugadores como individuos: demuestra conocimiento e interés por su vida fuera del terreno de juego.

"Dr. Wavne Halliwell. "La motivación en los deportes de equipo" (Apunts. 199.4).

Figura 1: Fútbol a la medida del niño, 2004, Horst Wein

A mediados de enero del 2020, en un canal de televisión deportivo (1), el presidente de la Asociación de Fútbol Argentino (AFA), Claudio Fabián Tapia, cuando le preguntaron por qué 
había elegido a Lionel Scaloni para ser el nuevo director técnico de la Selección Argentina, sin mucho preámbulo, colocó la "experiencia" como primer criterio de su elección. Señaló entonces que Scaloni tenía "mucha experiencia" como entrenador, pero, sobre todo, como jugador, lo cual justifica su nombramiento. En otro programa de televisión(2), en el año 2011, le preguntaron al ex presidente de la AFA Julio Humberto Grondona cuáles eran los valores que encontraba en Sergio Batista para que esté al frente del seleccionado nacional, lo primero que destacó Grondona fueron los conocidos antecedentes exitosos de Batista como jugador.

Estos son simples ejemplos, -tanto en lo que se denomina "fútbol formativo" como en el "fútbol profesional"- del modo en que se procede en el mundo futbolístico. Resulta evidente que la motorización de cualquier proyecto es jactada por la cuantificación vivencial sensible de las personas con el mundo y su cuota de exitismo.

\section{Los problemas de la experiencia}

Podríamos rastrear tres problemas que comparten la deshistorización del fútbol como práctica, repercutiendo una especie de "know how" en las personas implicadas en el fútbol: A) Una esencia estática y exitista; B) Una evolución natural y continua del conocimiento; C) El individualismo

A) El fútbol, en tanto esencia estática, se conoce en la repetición adhesiva de contactos sensibles, con lo cual, por un lado, el saber lo brinda la vivencia con el juego y por el otro, el fútbol en tanto como algo estático, nos conduce a decir que la actividad se repite. "El futbol será siempre antiguo. Porque no es ciencia que pueda enseñarse. Es imprevisto" (Panzeri, 2011, pág. 93).

La actividad consiste en una reproducción de los hechos. "Porque lo viví desde adentro y sé que se hace asi" se convierte en el fundamento del accionar, no hay lugar para cuestionamientos al desempeño. De esta manera, se pregona una continuidad de cierto status quo, cierta idea anticuaria de que todo pasado fue mejor, una conservación enmascarada contra todo intento de iniciativa novedosa.

Nuestra lucha por la responsabilidad se desarrolla contra un enmascarado. 
La máscara del adulto se llama <<experiencia >>. Siempre igual, inexpresiva, impenetrable. Este adulto ya lo ha vivido todo: la juventud, los ideales, las esperanzas, la mujer. $\mathrm{Y}$ todo era sólo una ilusión. A menudo estamos intimidados o amargados. Es posible que tenga razón el adulto. ¿Qué podemos nosotros contestarle? Todavía no tenemos experiencia. Vamos a intentar quitarle la máscara. ¿Qué ha experimentado este adulto? ¿Qué quiere demostrarnos? Sobre todo, una cosa: que también él fue joven, que también él quiso lo que nosotros queremos, que tampoco él creyó a sus padres, pero que también a él la vida le ha enseñado que sus padres tenían razón. El adulto sonríe con superioridad al decirnos que nos sucederá lo mismo a nosotros. De este modo desvaloriza de antemano los años que nosotros estamos viviendo, los convierte en la época de los dulces disparates juveniles, en la embriaguez infantil antes de la larga sobriedad de la vida seria. Así hablan los benévolos, los ilustrados. Conocemos a otros pedagogos cuya amargura ni siquiera nos concede los breves años de la «juventud»; ya quieren ponernos, serios y crueles, al entero servicio de la vida. Los dos desvalorizan nuestros años, hasta los destrozan. $Y$ cada vez nos invade en mayor grado este sentimiento: tu juventud sólo es una breve noche (en consecuencia, llénala de embriaguez); luego llegará la gran <<experiencía>>, los años de los compromisos, la pobreza de ideas y la falta de brío. Así es la vida. Eso nos dicen los adultos, así lo han experimentado ellos (Benjamin Walter, 2007, p. 54).

Aquí también lo problemático es el efecto exitista que produce este razonamiento, lo que se deriva de aquí es que, si en tu experiencia futbolística obtuviste buenos resultados, se deduce que contratándote vuelva a ocurrir el éxito, y para las experiencias por fuera de determinados resultados conlleva a la anulación o dificultad para dirigir, coordinar, presidir o simplemente tomar la palabra en algún ámbito futbolístico.

Es la vivencia, entonces, la que habilita el derecho a tomar la palabra ya que solo consiste en reproducir esa cuantificación vivencial, porque se entiende que el fútbol es siempre el mismo y discusión terminada. La cuestión de la experiencia planteada en estos términos, es 
decir, la objetividad del fútbol como un hecho que se repite es lo que en este deporte opera como un motor fundamental para impedir cualquier posible cambio, tanto en lo que respecta al juego en sentido estricto, como los sucesos políticos. Es decir, el cierre ante lo nuevo y la puesta en marcha de aquellos poderes más sutiles -pero no por eso menos importantes- que se ejercen en el mundo futbolístico (de clase, de género, de raza, de nepotismo, etc) para que nada cambie.

Lo cierto es que el conocimiento asociado a la práctica supone, además de la reserva masculina sobre su privilegio, un saber legitimado simplemente por haber pisado una cancha, en cualquiera de sus posiciones: de allí la reiteración de ex jugadores, ex técnicos y hasta ex árbitros transformados en voces autorizadas para hablar de lo que sea- ya no solo sobre sus prácticas específicas y especializadas, sino sobre cualquier otra, sobre cualquier cosa-. [...]. Desde el modo de parar la pelota hasta las soluciones a la violencia futbolística, todo se aprende en el mismo espacio: porque se trata de sabidurías puramente pragmáticas, accesibles por simple exposición a los hechos, a su vez irreductible a cualquier teoría. De allí que para ser director técnico haya que haber sido jugador, aun mediocre: en caso contrario, "no se conoce el vestuario". [...] Llevando esa afirmación al extremo, aguateros, kinesiólogos o camilleros podrían escribir solventemente libros de fútbol. O presidir comisiones investigadoras. O liderar paneles periodísticos conducidos por algunos buenos muchachos (Alabarces Pablo, 2014, pp.19-20).

B) El concepto mismo de evolución natural y continua. Una idea de evolución lineal en el conocimiento gracias a esa gran masa de hechos que vamos viviendo y nos van diciendo que hacer y qué no hacer. Una atemporalidad histórica, es decir, a medida que el tiempo avanza y nos topamos repetidamente con los hechos, por la adhesión de ellos es que vamos mejorando. Por eso es que la cuestión etaria de la gente que está a cargo de los planteles es un punto relevante aquí a considerar; y que nosotros vamos a tomarlo como un problema porque la experiencia "ya no está en dirección a la muerte, sino que retrocede hacia la infancia. En esa inversión del límite, así como en el pasaje 
de la primera a la tercera persona, debemos descifrar los rasgos de una nueva experiencia" (Agamben Giorgio, 2015, p.53).

La cantidad de tiempo que hayas jugado al fútbol o que hayas dirigido equipos reaparece constantemente como legitimación para enseñar fútbol; nos encontramos ante una idea aferrada sobre la experiencia como aquella acumulación continua y repetida de vivencias corporales que determinan conocimiento, o peor aún, que determinan que uno sepa enseñar sobre lo realizado, en nuestro caso, fútbol.

La experiencia en el sentido de aquella acumulación de vivencia individual -asociada no solo al tiempo que se practicó fútbol sino a la cantidad de años de la persona-, resulta un contenido indispensable a considerar a la hora de elegir un técnico o alguien encargado de enseñar fútbol. Es muy común la pregunta de un dirigente hacia alguien que quiera o que esté trabajando en el fútbol a cargo de algún grupo: ¿jugaste al fútbol? La segunda pregunta que siempre viene es: ¿dónde? Muchas veces se indaga en los resultados deportivos como garantía para verificar que se realiza bien la labor. Esto mismo ocurre en mayor medida para los planteles superiores: la idea de "tener mucho vestuario" garantiza la idoneidad del aspirante a director técnico, no el conocimiento acerca de conceptos y principios del juego.

A continuación, cito un fragmento de un documento analizado sobre un Director técnico especializado en formación de juveniles en la Argentina:

En primer lugar, el hecho de haber sido futbolista o campeón del mundo es un antecedente valido en algunos aspectos, pero no en todos... no quiere decir que porque yo jugué... Yo recuerdo que me llevaron a jugar a un equipo de una categoría inferior a Francia, [claro y claro], el presidente había leído que yo había jugado con Maradona, él me vio jugar a mí y me quería mandar de vuelta porque claro yo no era Maradona, había jugado con él. Un formador se nutra, [se nutre perdón], de las vivencias que tuvo. Tiene que definir muy claro cuál va a ser su filosofía de trabajo y en base a eso nutrirse de la gente que él considera que son sus referentes (Rossi Ruben, 2018). 
C) Otro problema que rastreamos en la exaltación de la vivencia, es su acento sobre el individualismo en el fútbol. Las decisiones de la gestión deportiva están siendo pensada desde la unidad, desde el individuo, o, mejor dicho, desde la gestión del individuo. El efecto a producir desde el entramado futbolístico, -y en donde la vivencia es constitutiva-, es individualizar la gestión para controlar el orden y la eficacia institucional. Detectar cual individualidad se ajusta del modo más eficaz al orden imperante y cual el descarte. De este modo, ante el surgimiento de alguna subjetividad distinta a lo esperado por dicho orden se facilita el juzgar y prescindir de dicho individuo, porque recordemos no priman las ideas, priman las identidades subjetivas que contienen potencial de vivencias. Se reconoce a la vivencia del individuo por sobre las ideas, por sobre el dominio discursivo sobre el fútbol. Un pliegue discursivo de la gestión que reduce la dimensión gregaria de lo humano en el estudio y vigilancia del individuo para sostener una organización con mayores niveles de control y un crecimiento de eficacia económica y política de unos pocos.

La gestión es la que está en la puerta de ingreso del fútbol, exigiendo y juzgando cuanto se adecua esa vivencia a la eficacia requerida. Como señala Michel Foucault en relación con la solicitud de la identidad: "No me pregunten quien soy, ni me pidan que permanezca invariable: es una moral de estado civil la que rige nuestra documentación" (2015, p.30). En esta línea de sentido, el fútbol no cesa de solicitar la documentación vivencial de las personas para operar en su funcionamiento. Esa documentación de la cuantificación vivencial del individuo y su rastreo exitista es la moralización de la identidad que el fútbol exige para habilitar la existencia en el mismo; cuando no se posee la documentación de la vivencia en el fútbol, se anula la discusión: quien no jugó, quien jugó poco y que encima no obtuvo buenos resultados asiste a mayores grados de una discriminación para hablar de fútbol, mucho más para ser director técnico. La vivencia es el discurso policial que constata la identidad de un individuo sobre su recorrido en el fútbol, la credencial para tomar la palabra, para acceder al ámbito anhelado. Cuanto más éxito se posee en esa vivencia la credencial aumenta en las puertas para ingresar hacia el plano de tomar la palabra, no es solo en relación al fútbol sino para todo tipo de asunto social. Aquí es donde se ve a ex jugadores hablando de política, de educación, de enseñanza, de seguridad, de cuestiones de género, de economía, etcétera. 


\section{Conclusiones}

Se puede ver fácil como ex jugadores pasan a ocupar cargos en la enseñanza del fútbol, en la gestión deportiva y también en "cargos políticos". Esa credencial vivencial para acceder al plano de la enseñanza o de la dirigencia, no es otra cosa que una alianza, una represión invisible del orden deportivo imperante. Un orden regido por los escalafones de un poder económico, patriarcal y racista que usa redituablemente a la práctica deportiva en beneficio de unos pocos; se contrata a la gente que la vivió desde adentro porque se entiende [o conviene entender] que el saber se absorbe con la vivencia sensible y porque el fútbol es un saber completo, todo inventado, no hay lugar para la modificación.

Por un lado, nos hallamos ante una concepción de saber "completo" que se repite, por otro, ante la idea de que la adquisición del saber funciona desde una sensibilidad continua, inmediata y adhesiva; por último, esa adquisición de saber se presenta como individual, del orden de lo privado para justamente vigilar, clasificar y elegir. De este modo, la vivencia resulta el alimento para este triple juego que sostiene una organización jerárquica y cristalizada en el establecimiento del deporte como mercancía; la vivencia, en este esquema, tiene su peso porque garantiza su orden imperante, lo sostiene y lo refuerza. Un problema del orden político en el fútbol, "la política como aquella trama de relaciones productora de efectos" (Colombani M. Cecilia, 2008, p 21) porque la vivencia no altera, reproduce el orden del fútbol, de este modo con su primacía en la vivencia- el fútbol disminuye las probabilidades de ser alterado tanto en su organización política como en sus enunciados epistémicos.

Aquí, para tomar distancia de éste proceder futbolístico nos amparamos en la importancia del corte con el continuo de los hechos. Walter Benjamin señala, en relación a esta cuestión: "Para que un trozo de pasado sea alcanzado por la actualidad, no ha de haber continuidad entre ellos." (2009, p 108). Para este corte, nos amparamos en la reconstrucción de conceptos sobre fútbol, porque justamente entendemos los conceptos como

uno de los modos de la información que todo ser vivo extrae de su medio y mediante la cual, a la inversa estructura ese medio. (...). Formar conceptos es una manera de vivir y no de matar la vida; es una manera de vivir con toda 
movilidad y no de inmovilizar la vida; es manifestar, entre los miles de millones de seres vivos que informan su medio y se informan a partir de él, una innovación que se juzgara, a gusto de cada uno, ínfima o considerable: un tipo muy particular de información (Foucault M. , 2012, pp. 264-265).

El fútbol -como toda práctica- siempre está habitado por pensamientos que lo moldean y lo configuran de acuerdo a determinados choques de fuerzas históricos relacionados directamente con el conjunto de juegos relacionales de una época. Las prácticas se enmarcan en los conjuntos discursivos, en el concepto como productor de vida y no en el conjunto de vivencias u observaciones prácticas. El equivocado énfasis en poner lo práctico por sobre lo teórico muchas veces ofrece una cantidad de obstáculos que la teoría no puede prever, para ello y en contraposición podemos tomar a la relación en términos de relevos:

Las relaciones teoría-practica son mucho más parciales y fragmentarias. Por una parte, una teoría siempre es local, relativa a un pequeño campo, aunque puede ser aplicada a otro, más o menos lejano. La relación de aplicación nunca es de semejanza. Por otra parte, desde que la teoría profundiza en su propio campo se enfrenta con obstáculos, muros, tropiezos que hacen necesarios que sea relevada por otro tipo de discurso (...). La práctica es un conjunto de relevos de un punto teórico a otro, y la teoría un relevo de una práctica a otra. Ninguna teoría puede desarrollarse sin encontrar una especie de muro y se precisa de la práctica para perforar ese muro (Foucault M. y., 1981, pp. 26-27).

Enlazar Erfahrung con una teoría del fútbol es un trabajo poco elaborado en el mundo futbolístico, justamente porque aquí lo que se pone en marcha es la cantidad de Erlebnis y no de Erfahrung. Esta última, es una experiencia audaz y colectiva, que más bien se ata a una concepción histórica para que justamente no haya algo que se repita sino más bien algo que permita que aparezcan hechos nuevos en el fútbol.

\section{Referencias bibliográficas}

Agamben, G. (2015). Infancia e historia. Ciudad Autonoma de Buenos Aires: Adriana Hidalgo. 
Alabarces, P. (2014). Héroes, machos y patriotas. el futbol entre la violencia y los medios . Buenos Aires: Aguilar.

Archetti , E. (2016). Masculinidades: fútbol, tango y polo en la Argentina. Ciudad Autónoma de Buenos Aires: CLUB HOUSE. Un sello de Ediciones Deldragón.

Benjamin, W. (2007). "Experiencia” (edición original de 1913) en Obras, libro II/vol. 1. Título original Gesammelte Schriften, edición de Rolf Tiedemann y Hermann Schweppenhäuser. Traducción de Jorge Navarro Pérez. Madrid: Abada Editores.

Benjamin, W. (2009). La dialéctica en suspenso. Fragmentos sobre la historia. Santiago: LOM.

Colombani, M. C. (2008). Foucault y lo político. Buenos Aires: Prometeo Libros.

Desio, H. (24 de Julio de 2017). La llave del Gol. FoxSports. Refundacion juvenil. ¿Cómo se trabaja en las nuevas selecciones? (R. Taquini, D. Latorre, D. Arcucci, \& J. Baravalle, Entrevistadores)

Foucault, M. (1992). Microfísica del poder. Madrid: La Piqueta.

Foucault, M. (2012). El poder, una bestia magnifica: Sobre el poder, la prision y la vida. Buenos Aires: Siglo veintiuno.

Foucault, M. (2015). La arqueología del saber. Buenos Aires: Siglo veintiuno.

Foucault, M. y. (1981). Un dialogo sobre el poder y otras conversaciones. Mandius.

Fusetti, P. (28 de abril de 2021). Fútbol argentino y ciencia. Una mirada sobre la experiencia y la enseñanza . Tesis para optar en el grado de Magíster en la Maestría en Deporte. La Plata, Buenos Aires, Argentina: UNLP.

Grondona, J. H. (14 de Enero de 2011). Esta Noche. Canal C5N. (G. Rozin, Entrevistador). Recuperado de: https://www.youtube.com/watch?v=FCXiixZIMYo

Moreira, V. (2018). Fútbol, modelos jurídicos y mercado: el dilema de los clubes en Sudamerica. Revista Crítica de Ciencias Sociales, 135-154. 
Panzeri, D. (2011). Fútbol. Dinámica de lo impensado. Madrid: Capitán Swing.

Rossi, R. (6 de Abril de 2018). La Llave del Gol. FoxSports. Las inferiores son para formar.

(R.Taquini, D. Latorre, D. Arcucci, \& J. Baravalle, Entrevistadores)

Staroselsky, T. (19 al 21 de Agosto de 2015). X Jornadas de Investigacion en Filosofia.

Consideraciones en torno al concepto de experiencia en Walter Benjamin. Ensenada, Buenos Aires, Argentina: Bibhuma.

Tapia, C. F. (15 de Enero de 2020). TyCSports Verano. (D. Diaz, Entrevistador). Recuperado de: https://www.youtube.com/watch?v=Dm_BGO7EmXg\&t=46s

Wein, H. (2004). Fútbol a la medida del niño. Volumen 1. Las Rozas: Gymnos

\section{Notas}

(1)TyCSports. "TyCSports Verano" (15 de Enero de 2020). Claudio Tapia: "Cosas que ví en Rusia me demostraron que Scaloni estaba preparado para conducir". Entrevistador Diego Diaz. Recuperado de: https://www.youtube.com/watch?v=Dm BGO7EmXg\&t=46s

(2)“C5N. "Esta noche”. Con Gerardo Rozin. (14 de enero de 2011)". Recuperado de: https://www.youtube.com/watch?v=FCXiixZIMYo 